\title{
Excitability changes in the visual cortex quantified with signal detection analysis
}

\author{
Manuel C. Olma, Antje Kraft, Jasper Roehmel, Kerstin Irlbacher and Stephan A. Brandt* \\ Department of Neurology, Charité Universitätsmedizin Berlin, Berlin, Germany
}

\begin{abstract}
Purpose: TDCS can increase excitability in the visual cortex. It is a matter of current debate if tDCS can improve visual performance. Promising parameters to measure detection sensitivity may be those of the signal detection theory (=SDT), as it allows differentiating between response bias and detection sensitivity changes. The measure of detection sensitivity can be used to predict actual performance under a wide variety of different response criteria.

Methods: Here we test if the SDT can quantify tDCS-induced effects in a visual contrast discrimination task in healthy subjects. Results: Anodal stimulation of the visual cortex improved performance, as calculated by detection sensitivity for stimuli presented in the center of the visual field. More peripheral locations in the visual field were unaffected by anodal stimulation. Cathodal stimulation and sham stimulation of the visual cortex had no consistent effect on detection sensitivity. The response bias was not affected by any type of stimulation.

Conclusions: Neuroplastic changes in the visual cortex induced by anodal tDCS can be measured by SDT, suggesting SDT could prospectively be a useful approach for monitoring restorative tDCS-effects on visual function in patients with central visual deficits.
\end{abstract}

Keywords: Transcranial direct current stimulation (tDCS), neuromodulation, visual psychophysical performance, signal detection theory, visual cortex

\section{Introduction}

Over the last decade, the manipulation of perceptual functions by noninvasive stimulation of the human brain has caused great interest. Transcranial direct current stimulation (tDCS) is a pain-free, non-invasive method for delivering weak polarizing electrical currents to the human cortex by means of two electrodes placed on the scalp (Nitsche and Paulus, 2000). It modulates neuronal excitability and activity in the human brain. Reliably, tDCS focally alters neuronal resting membrane potential, inducing prolonged changes in synaptic efficiency in the motor cortex: anodal tDCS

${ }^{*}$ Corresponding author: Stephan A. Brandt, Department of Neurology, Charité Universitätsmedizin Berlin, Charitéplatz 1, 10117 Berlin, Germany. Tel.: +49 30450660 111; Fax: +49 30450560 942; E-mail: stephan.brandt@ charite.de. enhances cortical excitability, while cathodal stimulation reduces it (Nitsche et al., 2008).

In the occipital lobe, tDCS is able to induce bidirectional excitability shifts of the primary visual cortex (V1). Importantly, changes in cortical excitability have been measured by an altered TMS phosphene threshold in V1 (Antal et al., 2003a). In the primary visual cortex, anodal tDCS diminished the threshold for static phosphenes. Cathodal stimulation resulted in an increase of the threshold for static phosphenes (Antal et al., 2003a).

In a prior study by Antal and colleagues (2001), it was demonstrated that $7 \mathrm{~min}$ of cathodal tDCS of the visual cortex induced a significant decrease in static and dynamic contrast sensitivities using a large $13^{\circ} \times 13^{\circ}$ Gabor patch. However, no effect of anodal tDCS on the sensitivity for temporal and spatial frequencies could be found. According to Antal and 
colleagues, a possible explanation is that an optimal functioning visual system, which processes an optimal stimulus, cannot be improved in its perceptual visual functions due to a ceiling effect (Antal et al., 2001).

Further studies revealed that tDCS is also able to neuromodulate the extrastriate visual cortical areas such as V5 effectively (Antal et al., 2004b,c). Specifically, anodal stimulation of V5 improved learning in a visuo-motor coordination task, while cathodal tDCS of the same region improved performance of the same task in an over-learned state. The effects of tDCS differ depending on which learning phase is affected by stimulation. While excitability enhancement during learning should increase the strength of task-relevant synaptic connections, the advantage of suppressing task-irrelevant connections may be greater during performance of an over-learned task (Antal et al., 2004b).

It seems that the duration of the after-effects is shorter in the visual cortex as compared to the motor system (Antal et al., 2006). Explanations for this discrepancy may be structural differences of the orientation, the neuronal architecture and the distribution of receptor densities of both cortices. Importantly, the distance from the scalp increases with eccentricity, i.e. cortical representations of more peripheral parts of the visual field, whereas the motor cortex is approximately equidistant from the scalp (Nitsche et al. 2008).

Comparability between tDCS-studies in the visual system is yet somewhat limited, as stimulation effects vary in dependence of stimulation parameter, such as stimulation site $(\mathrm{O} 1, \mathrm{O} 2, \mathrm{Oz}, \mathrm{V} 5)$, position of the reference electrode (posterior neckbase vs $\mathrm{Cz}$ ), measured parameter (VEP, motion perception, moving phosphene threshold, contrast sensitivity), (e.g. Antal et al., 2001, 2003a,b, 2004a,b,c; Accornero et al., 2007) and target eccentricity (Kraft et al., 2010). Thus, it is assumed that effects of tDCS in the visual system are strongly task dependent. However, the influence of changed excitability on standard psychophysical parameters (reaction time, error rate, detection sensitivity, threshold) is only poorly understood (Antal et al., 2008).

In a recent study, we have demonstrated that stimulating the visual cortex by anodal tDCS increases contrast sensitivity measured by threshold perimetry and this effect was dependent on eccentricity (Kraft et al., 2010). However, using threshold perimetry for measuring tDCS stimulation-effects necessitates an expensive ophthalmologic clinical device, which is usually not available in a standard clinical setting.
Therefore, an experimental psychophysical parameter that reflects tDCS-effects would simplify measurements in an experimental setup substantially.

The aim of our present study is to systematically investigate the impact of altered excitability in the visual cortex on the "signal detection theory" (SDT) parameters (Macmillan and Creelman, 2005), since it allows for quantification of the detection of signal against noise. The measure of detection sensitivity can be used to predict actual performance irrespective of different response criteria. Being able to test visual sensitivity independently of response bias makes the SDT a promising tool to monitor changes of excitability on visual psychophysical performance, and perspectively, to monitor restorative tDCS-effects on visual function in patients with visual deficits.

\section{Methods}

\subsection{Subjects}

Nine healthy right-handed subjects (age ranging from 22 to 29 years; 4 female) participated in the study with visual acuity better than 0.8 (without glasses). Subjects had no metallic implants or electrical devices, no evidence of neurological, psychiatric or ophthalmological pathology and were free of CNS-affecting medication or recreational drug use during the course of the experiments. All subjects gave written informed consent prior to their inclusion in the study. All subjects were paid for their participation. The study was performed in accordance with the ethical standards laid down in the 1964 Declaration of Helsinki and was approved by the local ethics committee.

\subsection{Stimulation experiment}

On each of three testing days, subjects were tested in a dimly lit room with a visual field testing distance of $60 \mathrm{~cm}$. For the psychophysical visual discrimination task we chose the modality of orientation because it is a psychophysically thoroughly-studied modality, which also permits the task difficulty to be increased simply by enhancing orientation similarity between stimuli. Furthermore, it has been shown that the modality of orientation is already processed in the early visual areas, such as V1 (Yacoub et al., 2008).

Subjects' task was to report as accurately as possible, whether a target or a distractor stimulus had been 


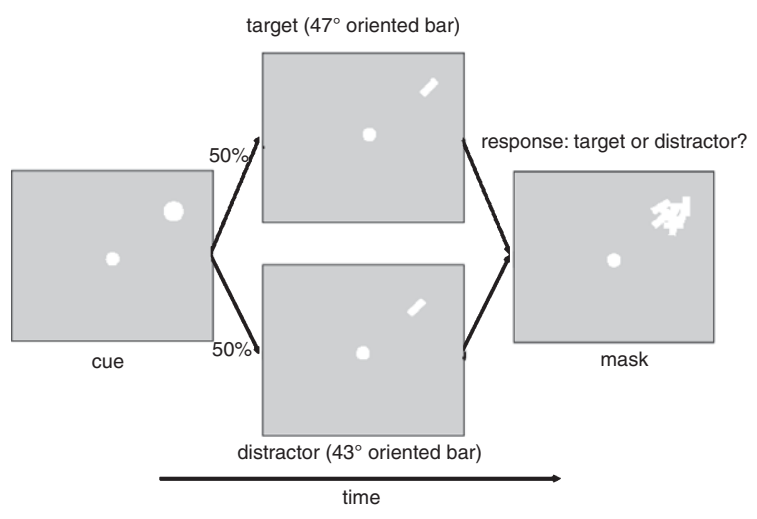

Fig. 1. Behavioral task with the three stimulus displays used. The left display depicts the location cue (displayed for $150 \mathrm{~ms}$ ), which could appear at one of five possible locations: central or at an eccentricity of $2^{\circ}$ on the bisection of each quadrant. The middle display shows the stimulus that had to be judged being either the target stimulus or the distractor stimulus. Target and distractor stimulus differed only in degrees of orientation. The orientation of the target stimulus was either $47^{\circ}$ and the orientation of the distractor stimulus was $43^{\circ}$ (as depicted) or vice versa. The presentation time of the stimulus was predetermined in a preceding control experiment calibrating individual performance to $80 \%$ correct with a mean presentation time of $143.1 \mathrm{~ms}$ (standard error $7.6 \mathrm{~ms}$ ) over all subjects and locations. The right display shows the visual masking stimulus, which was present until response was given by finger press.

displayed on a previously cued location (see Fig. 1). The stimuli were displayed on a laptop computer running Matlab 7.5.0 (The MathWorks). The visual stimuli were presented at an eccentricity of $2^{\circ}$ on one of the four diagonals of the display (i.e., at polar angles of $45^{\circ}, 135^{\circ}, 225^{\circ}$, and $315^{\circ}$ ) or at the centre of the display. The maximum eccentricity of the performed task was restricted to $2^{\circ}$, as it has been shown that visual discriminability decreases with eccentricity (Sally and Gurnsey, 2007). Moreover, cortical representations of peripheral eccentricities might be too distant from the scalp and thus from the occipital electrode for effective stimulation (Kraft et al., 2010).

The stimuli were light gray bars, which encompassed $1^{\circ} \times 0.17^{\circ}$ visual angle. The orientation of the stimuli was tilted in either $43^{\circ}$ or $47^{\circ}$. Before the experiment, subjects were instructed which orientation was assigned to the target and to the distractor stimulus. Thus, the orientation of the target stimulus was either $43^{\circ}$ and the orientation of the distractor stimulus was $47^{\circ}$ or vice versa. This assignment of orientation was counterbalanced over the group. Thus, target and distractor stimuli differed only in degrees of orientation.
The location-cue was a white filled circle subtending $1^{\circ}$ of visual angle, and was displayed at one of the five possible positions. Throughout the experiment a central fixation dot of $0.5^{\circ}$ of visual angle was presented.

Each trial began with the presentation of a locationcue for $150 \mathrm{~ms}$. Then, the discrimination stimulus was presented at the cued location for a predetermined duration until the mask appeared. The presentation time of the discrimination stimulus was determined in an additional calibration experiment (see below). The mask was displayed until response was given.

The structured backward mask consisted of ten randomly-oriented bars of same size and color as the discrimination stimulus, presented at the position of the discrimination stimulus. The mask covered the full size of the target and the distractor stimulus, respectively.

Responses were given on a response box by finger press, with one key for the left thumb and one key for the right thumb. The response mapping of the target and distractor stimulus were counterbalanced over subjects and sessions. The inter-trial interval lasted for a fixed duration of $1 \mathrm{~s}$.

Subjects had to fixate throughout the entire experiment in order to maintain a static and stable visual field on the retina. To control fixation, eye movements were recorded by an eye-movement recording device (high speed video eye tracker, $250 \mathrm{~Hz}$, Cambridge Research Systems). Trials in which eye movements occurred were excluded from further analysis.

\subsection{Calibration experiment}

On each testing day, the stimulation experiment was preceded by a calibration experiment. In the calibration experiment, the same task was used as in the stimulation experiment with the exception that the presentation time of the discrimination stimulus was variable in order to effectively manipulate the difficulty of the discrimination task. To this end, a stimulus onset asynchrony (SOA) was introduced. The SOA between discrimination stimulus and mask was individually calibrated for each subject for all five stimulus positions separately, to reach a psychophysical performance level of 70-80\% correct. For calibration, a threshold algorithm described by Kontsevich et al. (1999) was used, which implements a Bayesian adaptive method for threshold evaluation. This threshold algorithm has been shown to be robust to mistakes made at the beginning of the experiment. The algorithm was started in parallel and independently for all 
four quadrants and the central position. As a result, specific SOAs were determined within 300 valid trials, i.e., 60 trials per position. The mean SOA over all subjects and all positions was $143 \mathrm{~ms}$ with a standard error of $8 \mathrm{~ms}$.

In the calibration experiment, fixation was monitored by the same eye- movement recording device described above; invalid trials, in which central fixation was not maintained, were repeated. Thus, the total duration of the calibration varied between 11 and $22 \mathrm{~min}$.

\subsection{Experimental procedure}

Each session lasted approximately 2 hours and began with the SOA-calibration of the five positions. These SOA were used in the stimulation experiment before and after tDCS. The stimulation experiment lasted at least $15 \mathrm{~min}$ and ended after a multiple of 50 trials was completed (in order to test each position equally frequently).

Transcranial DC stimulation was applied for $15 \mathrm{~min}$ at intensity of $1.0 \mathrm{~mA}$. The current intensity was ramped over $15 \mathrm{~s}$ at the beginning and end of the stimulation. All subjects participated in three stimulation sessions, i.e., anodal, cathodal and sham stimulation.

The sequence of stimulation (sham, anodal, cathodal) was randomized and balanced across subjects. Given 6 possible permutations of the order of experiments, 3 of the possible permutations were used twice (6 subjects), the other 3 possible permutations were used only once ( 3 subjects). The interval between each of the three stimulation sessions was at least 3 days.

Transcranial direct current stimulation was carried out by an Eldith DC-stimulator (Neuroconn) in a double-blind design. Blinding of the examiner was realized by using the code list mode of the Eldith DC-stimulator. The code list included codes for sham and active stimulation. Stimulation codes were selected by a second person and not by the examiner. When the examiner entered a code number into the DC-stimulator, the subject received active or sham stimulation while the stimulation type was not displayed on the DC-stimulator. Since the DC-stimulator was able to stimulate in a bipolar fashion, both polarities (anodal and cathodal) could be applied without changing the set-up of the stimulation electrodes. Thus, examiner and subjects were uninformed about the stimulation type.
The reference polarizing scalp electrode, wrapped in an $8 \times 10 \mathrm{~cm}$ piece of saline-soaked synthetic sponge, was placed at the middle of the subject's head at $\mathrm{Cz}$. The occipital scalp polarizing electrode $(5 \times 5 \mathrm{~cm}$ sponge) was placed between the interhemispheric fissure and the left upper limit of the cerebellum, so that left occipital cortex was covered by the electrode.

TDCS can be focused by either enhancing the size of the reference or reducing the size of the stimulation electrode (Nitsche et al., 2007). Here we use a larger reference electrode. Due to the high intersubject variability of the precise location of these neuroanatomical structures, software for navigation (Exima, NBS-System, Nexstim, Helsinki, Finland) was used for the placement of the occipital electrode (see Fig. 2). To this end, structural three-dimensional magnetic resonance imaging (MRI) data sets were acquired from each participant with a 1.5 Tesla Magnetom Vision (Siemens, Erlangen, Germany). Structural data sets were acquired using a T1-weighted sagittal Magnetization Prepared - Rapid Gradient-Echo (MP-RAGE) sequence $\left(\mathrm{TR} / \mathrm{TE}=10 / 4 \mathrm{~ms}, \mathrm{FA}=12^{\circ}\right.$, $\mathrm{TI}=100 \mathrm{~ms}$, voxel size $=1 \mathrm{~mm} 3)$. The data sets were loaded into the personal computer-based navigation software system and were co-registered with landmarks of the subjects' head surface.

\subsection{Data analysis}

Performance was measured using signal detection analysis (Macmillan and Creelman, 2005). Detection sensitivity (d') scores were calculated by subtracting the $\mathrm{z}$-scores of the proportion of correct responses on the target trials (hit rate) from the proportion of incorrect responses on distractor trials (false alarm rate):

$$
\left.\mathrm{d}^{\prime}=\mathrm{z} \text { (hit rate }\right)-\mathrm{z} \text { (false alarm rate) }
$$

where the $\mathrm{z}$ scores represent the area under a standardized normal distributed curve (mean $=0$, standard deviation =1) for the hit rate and false alarm rate ratios. Response bias "criterion c" was calculated as:

$$
\mathrm{c}=-(\mathrm{z}(\text { hit rate })+\mathrm{z}(\text { false alarm rate })) / 2
$$

Statistical data analysis was conducted with the PASW software (Version 18.0). Psychophysical performance values (detection sensitivity " $d$ ", response bias "criterion c" and reaction times "rt") were entered in separate repeated measures three-way analyses of variance (ANOVA) with stimulation type (sham, 


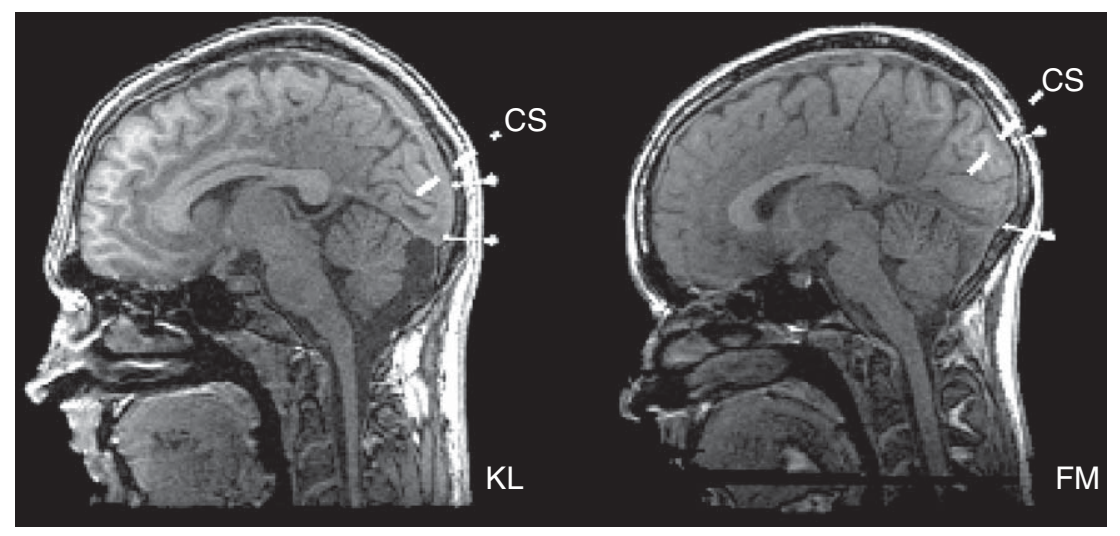

Fig. 2. MRI navigated positioning of the electrodes for stimulation of the visual cortex (navigated by Exima software, NBS-System, Nexstim, Helsinki, Finland). Parasagittal view of the left hemisphere of subject KL and FM. The occipital polarizing scalp electrode $(5 \times 5 \mathrm{~cm}$ sponge) was placed around the left sulcus calcarinus (CS, indicated by the white dashed line), so that the left occipital cortex was covered by the electrode. The gray arrows mark the projection of the upper and lower border of the electrode on the skull.

anodal, cathodal), time (pre, post) and position (upper left, upper right, lower left, lower right, central position). Additionally, the determined SOAs were entered in a regression analysis testing the variable day (day 1 , day 2, day 3 ) to evaluate potential learning effects across testing days. Where appropriate, degrees of freedom and $p$-values were corrected (GreenhouseGeisser) and planned paired $t$-tests were used to evaluate the differences between specific conditions.

\section{Results}

\subsection{Analysis of signal detection theory parameters}

Using the predetermined presentation times from the calibration experiment (see above) in the main experiment, $24.8 \%$ false alarms and $75.2 \%$ correct rejections were made across subjects when the distractor was presented. $16.3 \%$ misses and $83.7 \%$ hits occurred as a response to the presentation of the target.

The sensitivity parameter d' was entered in a repeated measures ANOVAs with factors stimulation type (sham, anodal, cathodal), time (pre, post) and location (upper left, upper right, lower left, lower right, central). The two-way interactions between the factors stimulation and location $[\mathrm{F}(8,64)=2.31, p=0.03]$, and between the factors time and location $[\mathrm{F}(4,32)=3.34, p=0.02]$ reached significance. Analysis revealed no significant main effects for the factors stimulation $[\mathrm{F}(1.2,9.5)=0.37$, $p>0.05]$, location $[\mathrm{F}(4,32)=0.12, p>0.05]$ and time $[\mathrm{F}(1,8)=0.75, p>0.05]$. The two-way interaction between stimulation $\times$ time $[\mathrm{F}(1.1,8.6)=1.42$, $p>0.05]$ and the three-way interaction between stimulation $\times$ location $\times$ time did not obtain significance $[\mathrm{F}(8,64)=1.47, p>0.05]$.

For planned paired $t$-test comparisons, data of the more peripheral locations was pooled in one condition. Planned t-test comparisons show that anodal stimulation increased d' at the central location (see Fig. 3) with a mean difference of d' before and after stimulation ( $\left.=\Delta d^{\prime}\right)$ of $0.41(p=0.02)$. Anodal stimulation did not affect d' at the more peripheral locations with $\Delta \mathrm{d}$ ' of $0.00(p>0.05)$. After cathodal or sham stimulation d' did not change significantly at any target location ( $\Delta$ d' cathodal: central -0.15 , peripheral $-0.06 ; \Delta d$ ' sham central -0.25 , peripheral -0.39$)(p>0.05)$.

As d' relates the hit rate and false alarm rate to each other, an increase of d' can either reflect more "hits" or less "false alarms" or both. In order to clarify which possibility is present in our data we analyzed the rate of hits and false alarms separately for the central position in the anodal stimulation condition, averaged over all subjects. Hit rate increased $4.3 \%$ and "false alarm" rate dropped $16.8 \%$ relative to the rates before anodal stimulation.

Since we stimulated the left hemisphere of the occipital cortex, we investigated whether there is any hemispheric benefit for targets that have been presented in the right hemifield. To this end, we conducted an 


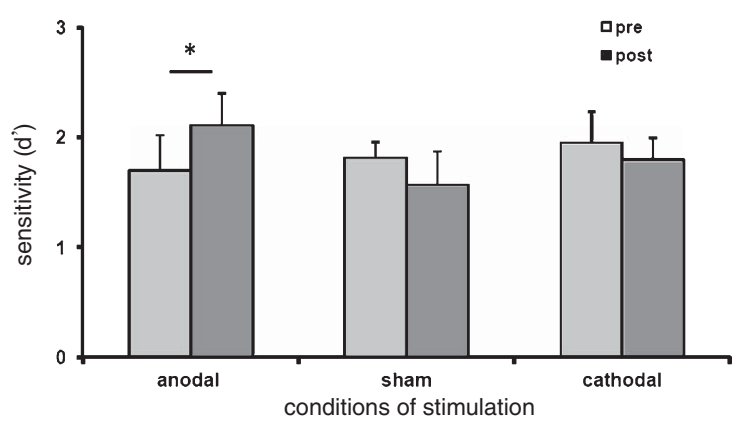

Fig. 3. Task performance. Sensitivity parameter d' depicted before (pre, light gray bars) and after (post, drak gray bars) anodal, cathodal and sham stimulation in the central condition of the discrimination task. *indicates significant difference $(p<0.05)$, as revealed by planned $t$-test comparisons. The error bars indicate the standard error.

ANOVA for d' for the factors stimulation type, time and hemifield (data of the central position was excluded for this anaylsis). There was no significant main effect or interaction effect of hemifield ( $p>0.05$, detailed results not reported).

In order to investigate whether DC-stimulation also changes the response bias, an ANOVA was conducted for "criterion c" for the factors stimulation type, time and location. There was no significant difference between stimulation $[\mathrm{F}(2,16)=1.68$, $p>0.05]$, time $[\mathrm{F}(1,8)=0.66, p>0.05]$ or location $[\mathrm{F}(4,32)=1.34, p>0.05]$. No interaction of the factors stimulation $\times$ time $[\mathrm{F}(2,16)=0.13, p>0.05]$, stimulation $\times$ location $[\mathrm{F}(8,64)=1.16, p>0.05]$, location $\times$ time $[\mathrm{F}(4,32)=0.22, p>0.05]$ or stimulation $\times$ time $\times$ location $[\mathrm{F}(8,64)=0.51, p>0.05]$ reached significance.

Since all subjects practiced the task repeatedly, learning effects need to be taken into account (see Kraft et al., 2010). However, in order to maintain a constant level of errors, in the present study, the presentation time of the stimulus was readjusted (see methods) before each stimulation session. We hypothesized that this procedure should balance possible learning effects. We entered the stimulus presentation time as dependent variable into a linear regression analysis. As expected, the stimulus presentation time significantly decreased in the course of the sessions $(p=0.02)$.

\subsection{Explorative analysis of reaction times}

Although the discrimination task was not speeded (subjects were not under time pressure to respond), we investigated exploratively whether there were also effects of tDCS on reaction times. To this end, extreme reaction times (below the $2.5 \%$ percentile or above the $97.5 \%$ percentile) were excluded from further analysis. The mean reaction times were $578 \mathrm{~ms}(\mathrm{sd}=186)$, indicating that subjects responded not only accurately but also relatively fast.

Reaction times were entered in a three-way ANOVA with factors stimulation type, time and location. A significant main effect for the factor "time" was obtained $[\mathrm{F}(1,8)=11.49, p=0.01]$ and a significant two-way interaction was found between the factors location $\times$ stimulation $[\mathrm{F}(8,64)=2.75, p=0.01]$. The two-way interaction between the factors location $\times$ time $[\mathrm{F}(4,32)=2.29, p=0.08]$ yielded marginal significance, whose meaningfulness could be corroborated by a high partial eta-squared of 0.22 and a low calculated power of $60.0 \%$. No significant main effects were revealed for the main factor stimulation $[\mathrm{F}(2,16)=0.98, p>0.05]$ or location $[\mathrm{F}(4,32)=1.82$, $p>0.05]$. Nor did the interactions between the factors stimulation $\times$ time $[\mathrm{F}(2,16)=1.29, p>0.05]$ or stimulation $\times$ time $\times$ location $[\mathrm{F}(8,64)=0.44, p>0.05]$ reach significance. Planned $t$-tests comparisons reflect that stimulation affected reaction times in the following way: both after anodal and sham stimulation of the visual cortex reaction times decreased significantly (for the left upper quadrant after anodal stimulation $(p=0.045)$, for all other positions $(p<0.01)$. After cathodal stimulation, reaction times increased in the central, left upper and right lower quadrant $(p<0.05)$; the right upper quadrant showed marginal significance $(p=0.08)$ and the lower left quadrant did not reach significance.

Since the parameter d' was affected for the central position by anodal stimulation we also compared the reaction times for the central position in the anodal stimulation condition for each response type (hits, correct rejections, false alarms and misses) separately. Reaction times of correct responses (hits, correct rejection) decreased significantly $(p=0.04)$ as opposed to incorrect responses (false alarm, miss). Here reaction times were not significantly changed after anodal stimulation $(p>0.05)$.

\section{Discussion}

The present study tested whether excitability changes in the visual cortex induced by transcranial direct current stimulation can be quantified with 
signal detection analysis parameters. By manipulating excitability via anodal tDCS applied to the occipital cortex, detection sensitivity could be increased in the central representation after stimulation in a discrimination task. Moreover, the increase of the sensitivity measure d' was reflected in an increase of the hit rate and the reduction of the false alarm rate indicating no criterion shift as expected. No improvement was seen in the more peripheral positions. Cathodal and sham stimulation did not alter detection sensitivity. The explorative analysis of reaction times showed that tDCS had an impact on reaction times. Subjects responded faster after both anodal and sham stimulation in all tested positions and tended to respond slower after cathodal stimulation in four out of five tested positions (the exception being the lower left quadrant).

The presence of an improvement in detection sensitivity cannot solely be ascribed to learning effects as each position of the visual field was tested equally frequent, thus was exposed to a comparable learning function. Otherwise one would expect that the sham condition, which reflects also a baseline learning condition, would yield the same pattern of improvement. Thus, the improvement of accuracy must be ascribed to the anodal tDCS, regardless of whether anodal stimulation influences the perceptual learning function.

With respect to the possibility that differential learning functions for the more peripheral positions were present, difficulty was readjusted for each position before each stimulation session by means of titrating the presentation time of the visual stimuli. Dockery and colleagues (2009) reported that the sequence of anodal or cathodal tDCS may interact with training phase (early vs. late) and possibly modify the effect of anodal or cathodal stimulation. This possible confounding effect has been addressed by balancing the sequence of anodal, cathodal and sham stimulation in the present study over subjects.

Another possible confounder is the anatomical variability of visual cortex in relation to the skull (Dougherty et al., 2003). Therefore, the usage of a skull based reference system (10-20 EEG-system), may lead to a broader cortical stimulation site, making stimulation effects less specific to the targeted cortical area. In order to address this issue we indentified the visual cortex using MRI-navigation.

The here presented positive effect of anodal tDCS on psychophysical performance agrees well with a recent tDCS study of our lab (Kraft et al., 2010), in which a visual detection task was performed using visual threshold perimetry. Only anodal tDCS was effective and thresholds for the central visual field positions were affected to a greater extent by anodal tDCS than thresholds at peripheral positions.

In this present study, anodal DC-stimulation yielded a dissociative pattern of results for discrimination stimuli in the periphery and the central location. Reaction times and d' are both positively affected by anodal stimulation in the centre; this is in contrast to the peripheral locations, where anodal stimulation decreased only reaction times effectively but not d'. A possible explanation could be that the stimuli at the peripheral positions had to be discriminated covertly, i.e., while maintaining central fixation. The central location could be performed overtly, i.e., eyes and the discrimination stimuli were at the same position. On the cortical level, the central visual field is represented overproportionally as compared to more peripheral locations of the visual field. Hence, more neurons are involved in encoding central stimulus information. Under the assumption that perceptual baseline noise is constant in the visual system, the stimulus signal-tonoise-ratio increases. This might be advantageous for further processing of the stimulus signal in the visual system.

Alternatively, this differential effect may result from an increased distance for more peripheral cortical representations of the visual field from the polarizing electrodes. Possibly, to impact on d' might necessitate a higher current density (putatively present in the representation of the central location) in comparison to impacting on reactions times. However, the interpretation of the reaction time results remains speculative, since the subjects' response was not speeded. A future study should test whether tDCS of the visual cortex leads to specific reaction time effects under speeded instruction.

In the study of Kraft and colleagues (2010) the occipital cortex was stimulated either on the left or the right hemisphere, but lateralized DC-stimulation affected both the contralateral and the ipsilateral visual hemifield. This is in line with our present study where no hemifield-effect was detectable for detection sensitivity or reaction times, e.g., reaction times were decreased by anodal stimulation over both hemifields by left occipital stimulation. In a realistic tDCS-head model, Sadleir and colleagues (2010) reported that high current densities were not only found directly under the electrodes but also occurred inter alia in proximate locations of the brain. 
Efficacy of anodal tDCS to enhance the excitability of the visual cortex has been demonstrated in other experiments (Antal et al., 2003a, b) measuring TMSinduced phosphene thresholds. However, Antal and colleagues reported no effect on the sensitivity for temporal and spatial frequencies after 7 min of anodal tDCS of the visual cortex (Antal et al., 2001). The absence of the expected aftereffects of anodal tDCS in this earlier experiment was assumed to be due to a ceiling effect as the visual system is highly optimized in perceptual performance. Differences in stimulus position and stimulus type could also be relevant factors as described in more detail in the introduction.

Our data, however, imply that performance of the visual system can be positively impacted by anodal tDCS in healthy subjects if the capacity of a specific visual perceptual function is at its limit. This effect might be particularly relevant and potentially beneficial for a visual system whose function has been limited by a brain lesion. Prospectively, SDT parameters might be adequate to monitor possible restorative effects of anodal tDCS on visual function in patients with central visual deficits. Along this clinical perspective, measuring d' is not based on the assumption that the motor system is unimpaired. If the motor system is affected by the patient's lesion, the motor impairment could confound the interpretation of reaction times, in contrast to d', which is independent from reaction times.

We demonstrate here for the first time that anodal stimulation of V1 can lead to a transient improvement of visual sensitivity during a discrimination task, measured by an established psychophysical parameter such as detection sensitivity, while response bias remained unaffected. TDCS modulates perceptual visual functions on a neural level, making it a promising tool in the field of neurorehabilitation. It is an open question whether patients with central visual field defects might benefit from anodal tDCS on the visual cortex, which needs to be tested in further clinically controlled studies.

\section{Acknowledgments}

We would like to thank the Else Kröner-FreseniusStiftung for funding this study. Furthermore, thanks to Richard A. Dargie for the helpful comments on the manuscript and English corrections.

\section{References}

Accornero, N., Li Voti, P., La Riccia, M. \& Gregori, B. (2007). Visual evoked potentials modulation during direct current cortical polarization. Exp Brain Res, 178(2), 261-266.

Antal, A., Nitsche, M.A. \& Paulus, W. (2001). External modulation of visual perception in humans. Neuroreport, 12(16), 3553-3555.

Antal, A., Kincses, T.Z., Nitsche, M.A. \& Paulus, W. (2003a). Manipulation of phosphene thresholds by transcranial direct current stimulation in man. Exp Brain Res, 150(3), 375-378.

Antal, A., Kincses, T.Z., Nitsche, M.A. \& Paulus, W. (2003b). Modulation of moving phosphene thresholds by transcranial direct current stimulation of V1 in human. Neuropsychologia, 41(13), 1802-1807.

Antal, A., Kincses, T.Z., Nitsche, M.A., Bartfai, O. \& Paulus, W. (2004a). Excitability changes induced in the human primary visual cortex by transcranial direct current stimulation: direct electrophysiological evidence. Invest Ophthalmol Vis Sci, 45(2), 702-707.

Antal, A., Nitsche, M.A., Kincses, T.Z., Kruse, W., Hoffmann, K.P. \& Paulus, W. (2004b). Facilitation of visuo-motor learning by transcranial direct current stimulation of the motor and extrastriate visual areas in humans. Eur J Neurosci, 19, 28882892.

Antal, A., Nitsche, M.A., Kruse, W., Kincses, T.Z., Hoffmann, K.P. \& Paulus, W. (2004c). Direct current stimulation over V5 enhances visuomotor coordination by improving motion perception in humans. J Cogn Neurosci, 16(4), 521-527.

Antal, A., Nitsche, M.A. \& Paulus, W. (2006). Transcranial direct current stimulation and the visual cortex. Brain Res Bull, 68(6), 459-463.

Antal, A., Terney, D., Poreisz, C. \& Paulus, W. (2007). Towards unravelling task-related modulations of neuroplastic changes induced in the human motor cortex. Eur J Neurosci, 26(9), 2687-2691.

Antal, A. \& Paulus, W. (2008). Transcranial direct current stimulation and visual perception. Perception, 37(3), 367-374.

Dockery, C.A., Hueckel-Wenig, R., Birbaumer, N. \& Plewnia, C. (2009). Enhancement of planning ability by transcranial direct current stimulation. J Neurosci, 29(22), 7271-7277.

Dougherty, R.F., Koch, V.M., Brewer, A.A., Fischer, B., Modersitzki, J. \& Wandell, B.A. (2003). Visual field representations and locations of visual areas V1/2/3 in human visual cortex. $J$ Vis, 3(10), 586-598.

Kontsevich, L.L. \& Tyler, C.W. (1999). Bayesian adaptive estimation of psychometric slope and threshold. Vision Res, 39(16), 27292737.

Kraft, A., Roehmel, J., Olma, M.C., Schmidt, S., Irlbacher, K. \& Brandt, S.A. (2010). Transcranial direct current stimulation affects visual perception measured by threshold perimetry. Exp Brain Res, 207(3-4), 283-290.

Macmillan, N.A. \& Creelman, C.D. (2005). Detection Theory: A User's Guide (2nd ed.), Lawrence Erlbaum Associates., Mahwah, N.J. 
Nitsche, M.A. \& Paulus, W. (2000). Excitability changes induced in the human motor cortex by weak transcranial direct current stimulation. J Physiol, 527, (Pt 3), 633-639.

Nitsche, M.A. \& Paulus, W. (2001). Sustained excitability elevations induced by transcranial DC motor cortex stimulation in humans. Neurology, 57, 1899-1901.

Nitsche, M.A., Nitsche, M.S., Klein, C.C., Tergau, F., Rothwell, J.C. \& Paulus, W. (2003). Level of action of cathodal DC polarisation induced inhibition of the human motor cortex. Clin Neurophysiol, 114, 600-604.

Nitsche, M.A., Doemkes, S., Karaköse, T., Antal, A., Liebetanz, D., Lang, N., Tergau, F. \& Paulus, W. (2007). Shaping the effects of transcranial direct current stimulation of the human motor cortex. J Neurophysiol, 97(4), 3109-3117.

Nitsche, M.A., Cohen, L.G., Wassermann, E.M., Priori, A., Lang, N., Antal, A., Paulus, W., Hummel, F., Boggio, P.S., Fregni, F.
\& Pascual-Leone, A. (2008). Transcranial direct current stimulation: State of the art 2008. Brain Stimul, 1(3), 206- 223.

Paulus, W. (2004). Outlasting excitability shifts induced by direct current stimulation of the human brain. Suppl Clin Neurophysiol, 57, 708-714.

Sadleir, R.J., Vannorsdall, T.D., Schretlen, D.J. \& Gordon, B. (2010). Transcranial direct current stimulation (tDCS) in a realistic head model. Neuroimage, 51(4), 1310-1318.

Sally, S.L. \& Gurnsey, R. (2007). Foveal and extra-foveal orientation discrimination. Exp Brain Res, 183(3), 351-360.

Yacoub, E., Harel, N. \& Uğurbil, K. (2008). High-field fMRI unveils orientation columns in humans. Proc Natl Acad Sci, 105, 1060710612. 